\title{
Inter-comparison of NIOSH and IMPROVE protocols for OC and EC determination: implications for inter-protocol data conversion
}

\author{
Cheng Wu ${ }^{1}$, X. H. Hilda Huang ${ }^{2}$, Wai Man $\mathrm{Ng}^{2}$, Stephen M. Griffith ${ }^{3}$, and Jian Zhen Yu $\mathbf{u}^{1,2,3}$ \\ ${ }^{1}$ Division of Environment, Hong Kong University of Science and Technology, Clear Water Bay, Hong Kong, China \\ ${ }^{2}$ Environmental Central Facility, Hong Kong University of Science and Technology, Clear Water Bay, Hong Kong, China \\ ${ }^{3}$ Department of Chemistry, Hong Kong University of Science and Technology, Hong Kong, China
}

Correspondence to: Jian Zhen Yu (jian.yu@ust.hk)

Received: 4 April 2016 - Published in Atmos. Meas. Tech. Discuss.: 24 May 2016

Revised: 26 August 2016 - Accepted: 28 August 2016 - Published: 14 September 2016

\begin{abstract}
Organic carbon (OC) and elemental carbon (EC) are operationally defined by analytical methods. As a result, OC and EC measurements are protocol dependent, leading to uncertainties in their quantification. In this study, more than 1300 Hong Kong samples were analyzed using both National Institute for Occupational Safety and Health (NIOSH) thermal optical transmittance (TOT) and Interagency Monitoring of Protected Visual Environment (IMPROVE) thermal optical reflectance (TOR) protocols to explore the cause of EC disagreement between the two protocols. EC discrepancy mainly $(83 \%)$ arises from a difference in peak inert mode temperature, which determines the allocation of $\mathrm{OC}_{\mathrm{NSH}}$, while the rest $(17 \%)$ is attributed to a difference in the optical method (transmittance vs. reflectance) applied for the charring correction. Evidence shows that the magnitude of the EC discrepancy is positively correlated with the intensity of the biomass burning signal, whereby biomass burning increases the fraction of $\mathrm{OC}_{\mathrm{NSH}}$ and widens the disagreement in the inter-protocol EC determination. It is also found that the EC discrepancy is positively correlated with the abundance of metal oxide in the samples. Two approaches (M1 and M2) that translate NIOSH TOT OC and EC data into IMPROVE TOR OC and EC data are proposed. M1 uses direct relationship between $\mathrm{EC}_{\mathrm{NSH}}$ _TOT and $\mathrm{EC}_{\mathrm{IMP} \_\mathrm{TOR}}$ for reconstruction:
\end{abstract}

M1 : $\mathrm{EC}_{\mathrm{IMP} \_\mathrm{TOR}}=a \times \mathrm{EC}_{\mathrm{NSH} \_\mathrm{TOT}}+b$;

while $\mathrm{M} 2$ deconstructs $\mathrm{EC}_{\mathrm{IMP}}$ TOR into several terms based on analysis principles and applies regression only on the un- known terms:

M2: EC IMP_TOR $_{\text {II }}=$ $\mathrm{AEC}_{\mathrm{NSH}}+\mathrm{OC}_{\mathrm{NSH}}-\left(a \times \mathrm{PC}_{\mathrm{NSH}} \_\mathrm{TOR}+b\right)$,

where $\mathrm{AEC}_{\mathrm{NSH}}$, apparent $\mathrm{EC}$ by the NIOSH protocol, is the carbon that evolves in the $\mathrm{He}-\mathrm{O}_{2}$ analysis stage, $\mathrm{OC} 4_{\mathrm{NSH}}$ is the carbon that evolves at the fourth temperature step of the pure helium analysis stage of NIOSH, and $\mathrm{PC}_{\mathrm{NSH}}$ TOR is the pyrolyzed carbon as determined by the NIOSH protocol. The implementation of M1 to all urban site data (without considering seasonal specificity) yields the following equation:

M1 $($ urban data $): \mathrm{EC}_{\mathrm{IMP} \_\mathrm{TOR}}=2.20 \times \mathrm{EC}_{\text {NSH_TOT }}-0.05$.

While both $\mathrm{M} 1$ and $\mathrm{M} 2$ are acceptable, M2 with sitespecific parameters provides the best reconstruction performance. Secondary OC (SOC) estimation using OC and EC by the two protocols is compared. An analysis of the usability of reconstructed $\mathrm{EC}_{\mathrm{IMP}}$ TOR and $\mathrm{OC}_{\mathrm{IMP}}$ TOR suggests that the reconstructed values are not suitable for SOC estimation due to the poor reconstruction of the OC / EC ratio.

\section{Introduction}

Carbonaceous aerosols are one of the major components of fine particulate matter $\left(\mathrm{PM}_{2.5}\right)$ in urbanized areas as a result of intense anthropogenic emissions. Carbonaceous aerosols consist of three categories: organic carbon (OC), elemental carbon (EC), and carbonate carbon (CC). OC can be either 
primary or secondary in origin, but EC is exclusively from primary emission. CC is only abundant in regions affected by mineral dust outflow and is negligible in other areas. OC and EC not only contribute to the overall $\mathrm{PM}_{2.5}$ load, but these components have specific public health concerns because of their interactions with the human body (Dou et al., 2015; Shi et al., 2015), and they significantly contribute to visibility degradation (Malm et al., 1994) and climate forcing (Bond et al., 2011).

Differentiating OC and EC is still challenging due to their complex chemical structure and optical properties. The most widely used technique to separate $\mathrm{OC}$ and $\mathrm{EC}$ is thermal optical analysis (TOA), which involves volatilizing the OC from a substrate while increasing the temperature by steps in an inert pure-helium atmosphere followed by combusting EC component in an oxygenated $\mathrm{He}$ atmosphere. A correction for charred OC (pyrolysis carbon, PC) in the inert stage relies on continuous monitoring of laser transmittance or reflectance of the filter. However, the separation of OC and EC in TOA is operationally defined due to the lack of widely accepted reference materials for calibration. A variety of TOA protocols are used by different research groups and monitoring networks (Watson et al., 2005). Among the TOA protocols, National Institute for Occupational Safety and Health (NIOSH; Birch and Cary, 1996) and Interagency Monitoring of Protected Visual Environment (IMPROVE; Chow et al., 1993) are most widely applied, which differ in their temperature ramping, step duration, and optical correction schemes (Table S1 in the Supplement). It is worth noting that the NIOSH protocol only outlines the necessary analysis principle for operation without specifying detailed technical parameters. Therefore, a number of NIOSH-type protocols exist in the literature (Watson et al., 2005), with the peak inert mode temperatures (PIMTs) varied from 850 to $940^{\circ} \mathrm{C}$.

Previous studies suggest that total carbon (TC), which is the sum of OC and EC, agrees very well (Chow et al., 2001) between the two protocols, but measured EC differs by a factor of 2-10, depending on the source and aging of the samples (Chow et al., 2001; Cheng et al., 2014). The EC discrepancy between NIOSH and IMPROVE mainly arises from the temperature ramping regime and the charring correction. The PIMT in NIOSH $\left(870^{\circ} \mathrm{C}\right)$ is much higher than in IMPROVE $\left(550^{\circ} \mathrm{C}\right)$. Thus, NIOSH may be subject to premature EC evolution (i.e., underestimation of EC), but IMPROVE may overestimate EC following incomplete OC evolution in the inert atmosphere (Piazzalunga et al., 2011). Since the optimal PIMT could vary between samples, a universal PIMT does not exist to avoid both of these biases (Subramanian et al., 2006). It should be noted that the residence time is different from sample to sample as the IMPROVE protocol only advances temperature to the next step until a well-defined carbon peak has evolved. In addition, IMPROVE uses a laser reflectance signal to perform the charring correction (TOR, thermal optical reflectance), while NIOSH adopts a laser transmittance for charring correction (TOT, thermal optical transmittance). Correction by reflectance only accounts for charring at the filter surface (Chow et al., 2004), while the transmittance correction considers charring throughout the filter, leading to a discrepancy in reporting PC.

The Pearl River Delta (PRD) is one of the most developed areas in China and home to the biggest city clusters in the world (World Bank, 2015). Air pollution issues have arisen from the economic bloom since the 1980s and pose a threat to public health (Tie et al., 2009). Although it is one of the biggest cites in the PRD, Hong Kong lacked an air quality objective regarding $\mathrm{PM}_{2.5}$ until January 2014. To better understand the variability of chemical compositions of $\mathrm{PM}_{2.5}$, the Hong Kong Environmental Protection Department of the Hong Kong Special Administration Region (HKEPD) has established a regular $\mathrm{PM}_{2.5}$ speciation monitoring program since 2011, including six monitoring sites, covering both suburban and urban conditions. The samples collected in the 3-year period 2011-2013 were analyzed by the Environmental Central Facility at the Hong Kong University of Science and Technology. These samples have been analyzed by both NIOSH TOT and IMPROVE TOR protocols, providing a unique opportunity to explore the OC and EC determination dependency on analysis protocols, which is the focus of this study. This study aims to answer the following questions: (1) what is the magnitude of the EC disagreement between the two protocols for Hong Kong samples? (2) What are the contributing factors, and how do they affect the EC discrepancy? (3) Is it feasible to perform OC and EC data inter-protocol conversion? (4) If yes, can the results be further used for secondary organic carbon (SOC) estimation?

\section{Methods}

\subsection{Sample description}

One $24 \mathrm{~h} \mathrm{PM}_{2.5}$ sample (from midnight to midnight) was programmed and collected every six days from January 2011 to December 2013 at six air quality monitoring sites (AQMS) in Hong Kong. The monitoring stations include Mong Kok (MK) just beside a busy road, Central/Western (CW), Tsuen Wan (TW), Tung Chung (TC) and Yuen Long (YL) at several meters above ground in urban areas in Hong Kong, and Clear Water Bay (WB) in a suburban area, as shown in Fig. S1 in the Supplement. Partisol samplers (Rupprecht \& Patashnick (now Thermo Fisher Scientific), Model 2025, Albany, NY) equipped with a Very Sharp Cut Cyclone (VSCC, BGI, Waltham, MA, USA) and operating at a flow rate of 16.7 $\mathrm{L} \mathrm{min}^{-1}$ were deployed at each AQMS. Two types of filter substrate were used: quartz filter (Pall, $47 \mathrm{~mm}$ 2500QAT-UP-47, Ann Arbor, MI, USA) and Teflon filter (Whatman, PTFE, $46.2 \mathrm{~mm}$ with a support ring, Clifton, NJ, USA). Sample filters were retrieved within $24 \mathrm{~h}$ and stored in Petri dishes sealed with parafilm under freezing temperatures. 
(a) $\mathrm{NIOSH}$
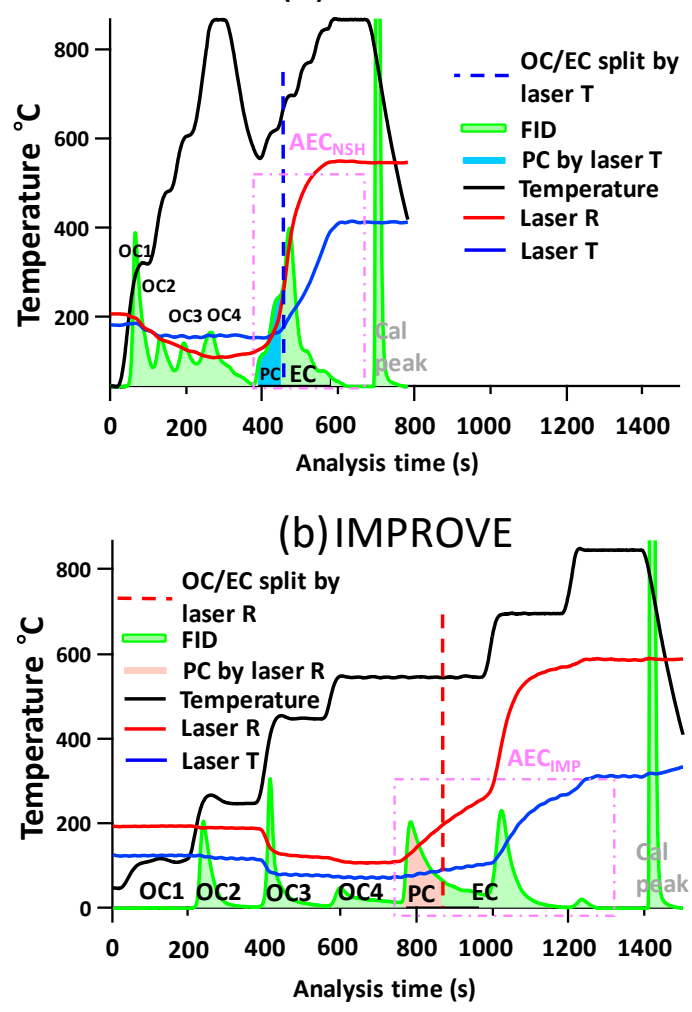

Figure 1. Thermograph of typical thermal optical analysis (sample CW20130118) using a Sunset carbon analyzer. (a) NIOSH protocol; (b) IMPROVE protocol (FID: flame ionization detector signal; PC: pyrolysis carbon; AEC: apparent EC, which is the sum of all the EC fractions before correcting for PC; temperature: oven temperature during analysis; Laser T: laser transmittance signal; Laser $\mathrm{R}$ : laser reflectance signal; Cal peak: calibration peak at the end of each analysis).

\subsection{Sample analysis}

Chemical analysis methods were described in detail by Huang et al. (2014), so only a brief description is given here. Teflon filters were first used for gravimetric analysis for $\mathrm{PM}_{2.5}$ mass concentrations using a microbalance (Sartorius, MC-5, Göttingen, Germany) in a temperature- and relativehumidity-controlled room, and then were used for elemental analysis (for more than 40 elements with atomic number ranging from 11 to 92) with an X-ray fluorescence (XRF) spectrometer (PANalytical, Epsilon 5, Almelo, the Netherlands). Quartz filters were analyzed by ion chromatography (Dionex, ICS-1000, Sunnyvale, CA, USA) and by TOA using a Sunset Laboratory analyzer (Tigard, OR, USA). All the OCEC samples were analyzed on the same Sunset analyzer using both NIOSH and IMPROVE protocols. Detailed temperature programs of the two protocols are shown in Table S1, and example analysis thermographs are shown in Fig. 1. The carbon analyzer is capable of performing both

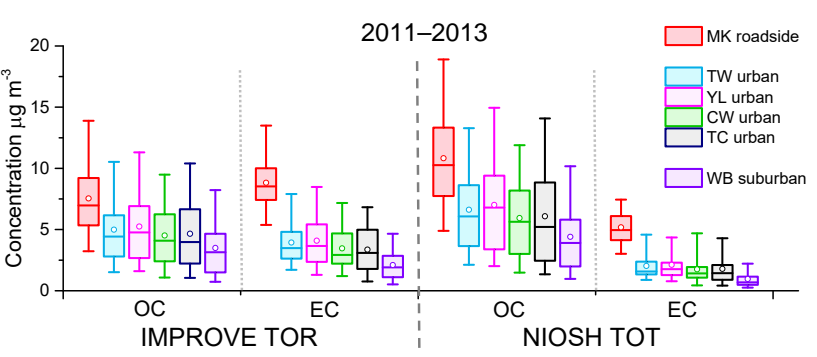

Figure 2. Three-year distributions of OC and EC concentrations by IMPROVE TOR and NIOSH TOT protocols for six sites in Hong Kong. The symbols in the boxplots represent the average (open circles), median (interior lines), 75th and the 25th percentile (box boundaries), and 95 th and 5th percentile (whiskers).

laser transmittance and reflectance charring corrections; thus both TOT and TOR results can be obtained for each protocol temperature program. As a result, four sets of analysis data are obtained and used for investigation of $\mathrm{OC}$ and EC determination dependency on analysis protocols in this study. The four sets of data are denoted as NIOSH TOT, NIOSH TOR, IMPROVE TOT, and IMPROVE TOR, with NIOSH and IMPROVE representing their respective temperature program and TOT and TOR representing the mean of charring correction based on laser transmittance and reflectance, respectively. It should be noted that NIOSH TOT and IMPROVE TOR data represent data by the two protocols, while the other two sets of data are usually not reported in EC and OC analysis. The concentrations of watersoluble organic carbon (WSOC) and three sugar compounds (levoglucosan, mannosan, and galactosan) were available for 2013 WB samples from a separate project. WSOC concentrations were measured by a TOC analyzer (Shimadzu TOC$\mathrm{V}_{\mathrm{CPH}}$, Japan) (Kuang et al., 2015). The sugars were analyzed by high-performance anion-exchange chromatography (HPAEC) with a pulsed amperometric detection (PAD) method (Engling et al., 2006).

\subsection{Quality assurance/quality control of OCEC data}

Since OC and EC are operationally defined and lack reference materials, external calibration is only performed for TC on a biweekly basis using sucrose solutions (Wu et al., 2012). Duplication analysis covering $14 \%$ of the total samples was conducted for quality control purposes. TC by the two protocols (NIOSH and IMPROVE) agrees very well as evidenced by the unity regression slope (Fig. S2a, slope $\left.=0.99, R^{2}=0.99\right)$ and sharp frequency distribution of NIOSH TC / IMPROVE TC ratios (Fig. S2b). Nevertheless, a small number of extreme data remain. The following criteria are used during the data processing to screen out the suspicious data: $0.1<\mathrm{OC} / \mathrm{EC}<40 ; 0.5<\mathrm{TC}_{\mathrm{NSH}} / \mathrm{TC}_{\mathrm{IMP}}<2$. After screening, a total of 1398 OCEC data points are used in this study. 
Table 1. Ambient mean concentrations $\left(\mu \mathrm{g} \mathrm{m}^{-3}\right.$ ) of OC and EC for six sites in Hong Kong by IMPROVE TOR and NIOSH TOT protocols.

\begin{tabular}{|c|c|c|c|c|c|c|c|c|c|}
\hline \multirow[t]{3}{*}{ Site } & \multirow[t]{3}{*}{$\begin{array}{l}\text { Analysis } \\
\text { protocol }\end{array}$} & & \multirow{3}{*}{$\begin{array}{r}\begin{array}{r}\text { Chow et } \\
\text { al. }(2002)^{\mathrm{b}} \\
2001^{\mathrm{a}}\end{array} \\
\end{array}$} & \multirow{3}{*}{$\begin{array}{r}\begin{array}{r}\text { Chow et } \\
\text { al. }(2006)^{\mathrm{b}}\end{array} \\
2005\end{array}$} & \multirow{3}{*}{$\begin{array}{r}\begin{array}{r}\text { Chow et } \\
\text { al. }(2010)^{\text {b }}\end{array} \\
2009\end{array}$} & \multicolumn{4}{|c|}{ Current study } \\
\hline & & & & & & 2011 & 2012 & 2013 & 3-year average \\
\hline & & & & & & \multicolumn{4}{|c|}{ (Mean \pm one standard deviation) } \\
\hline \multirow[t]{4}{*}{ MK } & IMPROVE & $\mathrm{OC}$ & 16.64 & 11.17 & 6.26 & $8.09 \pm 3.67$ & $6.94 \pm 2.55$ & $6.92 \pm 3.36$ & $7.33 \pm 3.28$ \\
\hline & TOR & $\mathrm{EC}$ & 20.29 & 14.11 & 10.66 & $8.48 \pm 2.08$ & $9.21 \pm 2.74$ & $9.42 \pm 1.89$ & $9.03 \pm 2.27$ \\
\hline & NIOSH & $\mathrm{OC}$ & & & & $11.36 \pm 4.26$ & $10.24 \pm 3.94$ & $10.51 \pm 4.63$ & $10.72 \pm 4.3$ \\
\hline & TOT & $\mathrm{EC}$ & & & & $4.86 \pm 1.47$ & $5.53 \pm 1.42$ & $5.35 \pm 1.78$ & $5.24 \pm 1.59$ \\
\hline \multirow[t]{4}{*}{ TW } & IMPROVE & $\mathrm{OC}$ & 8.69 & 6.93 & 4.38 & $5.44 \pm 3.35$ & $4.5 \pm 2.4$ & $4.86 \pm 3.47$ & $4.94 \pm 3.14$ \\
\hline & TOR & $\mathrm{EC}$ & 5.37 & 6.25 & 3.76 & $4.24 \pm 1.81$ & $3.62 \pm 1.99$ & $4.01 \pm 1.71$ & $3.97 \pm 1.84$ \\
\hline & NIOSH & $\mathrm{OC}$ & & & & $7.37 \pm 4.05$ & $6.1 \pm 3.33$ & $6.79 \pm 4.46$ & $6.77 \pm 4.01$ \\
\hline & TOT & $\mathrm{EC}$ & & & & $1.95 \pm 0.93$ & $1.76 \pm 0.91$ & $1.91 \pm 0.87$ & $1.88 \pm 0.9$ \\
\hline \multirow[t]{4}{*}{ YL } & TOR OC & $\mathrm{OC}$ & & 7.23 & 4.83 & $5.62 \pm 3.56$ & $4.77 \pm 3.02$ & $4.92 \pm 4.05$ & $5.16 \pm 3.63$ \\
\hline & TOR EC & $\mathrm{EC}$ & & 6.19 & 3.48 & $4.56 \pm 2.48$ & $3.69 \pm 1.8$ & $3.92 \pm 1.87$ & $4.08 \pm 2.1$ \\
\hline & TOT OC & $\mathrm{OC}$ & & & & $7.92 \pm 4.69$ & $6.33 \pm 3.94$ & $6.88 \pm 4.92$ & $7.12 \pm 4.62$ \\
\hline & TOT EC & $\mathrm{EC}$ & & & & $1.89 \pm 0.9$ & $1.79 \pm 0.91$ & $1.95 \pm 1.12$ & $1.88 \pm 0.98$ \\
\hline \multirow[t]{4}{*}{$\mathrm{CW}$} & IMPROVE & $\mathrm{OC}$ & & & & $4.92 \pm 2.89$ & $4.12 \pm 2.64$ & $4.37 \pm 3.33$ & $4.48 \pm 2.98$ \\
\hline & TOR & $\mathrm{EC}$ & & & & $3.71 \pm 1.75$ & $3.24 \pm 1.94$ & $3.48 \pm 1.69$ & $3.48 \pm 1.79$ \\
\hline & NIOSH & $\mathrm{OC}$ & & & & $6.55 \pm 3.55$ & $5.55 \pm 3.27$ & $6.2 \pm 4.02$ & $6.12 \pm 3.64$ \\
\hline & TOT & $\mathrm{EC}$ & & & & $1.63 \pm 0.82$ & $1.54 \pm 1.03$ & $1.54 \pm 0.95$ & $1.57 \pm 0.93$ \\
\hline \multirow[t]{4}{*}{$\mathrm{TC}$} & IMPROVE & $\mathrm{OC}$ & & & & $5.13 \pm 3.69$ & $4.17 \pm 2.68$ & $4.27 \pm 4.23$ & $4.53 \pm 3.63$ \\
\hline & TOR & $\mathrm{EC}$ & & & & $3.65 \pm 2.3$ & $3.1 \pm 1.71$ & $3.37 \pm 2.14$ & $3.38 \pm 2.08$ \\
\hline & NIOSH & $\mathrm{OC}$ & & & & $6.88 \pm 4.74$ & $5.48 \pm 3.37$ & $6.03 \pm 5.39$ & $6.15 \pm 4.63$ \\
\hline & TOT & $\mathrm{EC}$ & & & & $1.53 \pm 0.91$ & $1.55 \pm 0.87$ & $1.46 \pm 0.91$ & $1.51 \pm 0.89$ \\
\hline \multirow[t]{4}{*}{ WB } & IMPROVE & $\mathrm{OC}$ & & & & $3.91 \pm 2.62$ & $3.07 \pm 2$ & $3.37 \pm 3.13$ & $3.46 \pm 2.65$ \\
\hline & TOR & $\mathrm{EC}$ & & & & $2.43 \pm 1.42$ & $1.81 \pm 1.2$ & $1.96 \pm 1.39$ & $2.08 \pm 1.37$ \\
\hline & NIOSH & $\mathrm{OC}$ & & & & $5.07 \pm 3.33$ & $3.91 \pm 2.53$ & $4.62 \pm 3.93$ & $4.55 \pm 3.36$ \\
\hline & TOT & $\mathrm{EC}$ & & & & $0.86 \pm 0.5$ & $0.72 \pm 0.44$ & $0.67 \pm 0.58$ & $0.75 \pm 0.52$ \\
\hline
\end{tabular}

${ }^{\text {a }}$ November 2000-October 2001; ${ }^{\text {b }}$ studies using DRI2001 carbon analyzer.

\section{Results and discussion}

\subsection{Ambient PM $\mathrm{PM}_{2.5} \mathrm{OC}$ and EC concentrations}

The 3-year distribution of OC and EC concentrations is shown in Fig. 2, where a clear spatial gradient can be seen from the roadside site to the urban sites and suburban site. $\mathrm{OC}$ and EC levels at the MK roadside site are a factor of 2 higher for both protocols compared to the urban sites. Annual average concentrations and standard deviations for the five sites are listed in Table 1. Compared to samples collected at the MK and TW sites in November 2000-December 2001 (Chow et al., 2002), both OC and EC 3-year annual average concentrations observed in this study are lower by a factor of 1.4-2.3. At the TW site, TOR OC decreased from 8.69

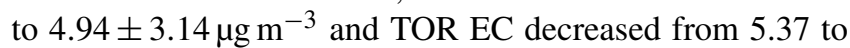
$3.97 \pm 1.84 \mu \mathrm{g} \mathrm{m}^{-3}$. The reduction is more pronounced at the MK roadside site, where TOR OC decreased from 16.64 to
$7.33 \pm 3.28 \mu \mathrm{g} \mathrm{m}^{-3}$ and TOR EC decreased from 20.29 to $9.03 \pm 2.27 \mu \mathrm{g} \mathrm{m}^{-3}$ (Chow et al., 2002).

\subsection{NIOSH and IMPROVE comparison for $\mathrm{OC}$ and EC determination}

The data discussed in this section use the unit of $\mu \mathrm{g} \mathrm{cm}^{-2}$ because the inter-protocol comparison focuses on the analytical aspect of OC / EC analysis, which is more associated with filter loading than air concentration. For data analysis involving linear regression, ordinary least-squares (OLS) regression is not suitable due to its error-free assumption in independent variables, which is unrealistic for OCEC data (Saylor et al., 2006). Weighted orthogonal distance regression (WODR) is employed in this study to account for the comparable degrees of uncertainty in both $x$ and $y$ (Boggs et al., 1989). As mentioned earlier, the difference in the peak inert mode temperature for IMPROVE $\left(550^{\circ} \mathrm{C}\right)$ and NIOSH $\left(870^{\circ} \mathrm{C}\right)$ is an important distinguishing factor between the 

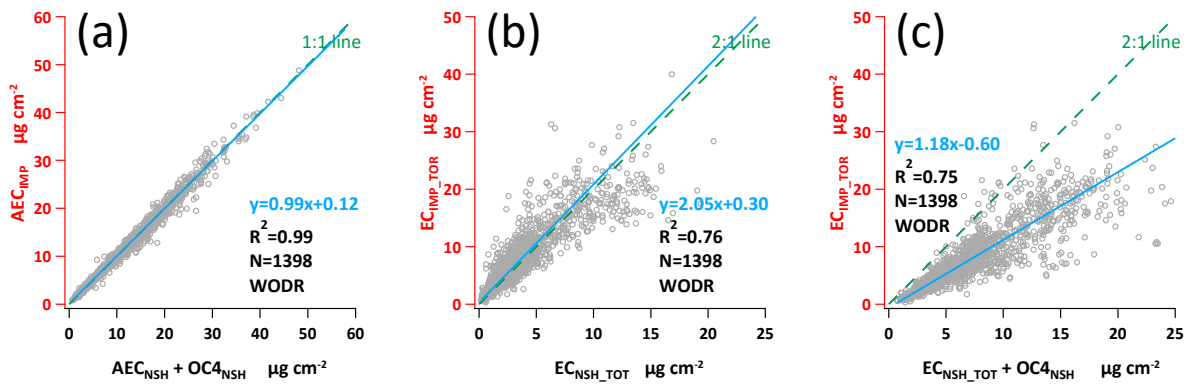

Figure 3. Comparison of different carbon fractions. (a) Relationship of IMPROVE apparent EC (AEC $\mathrm{IMP}_{\mathrm{IMP}}$ sum of EC $1_{\mathrm{IMP}}$ to EC $3_{\mathrm{IMP}}$ ) and the sum of $\mathrm{NIOSH}$ apparent $\mathrm{EC}\left(\mathrm{AEC}_{\mathrm{NSH}}\right.$, sum of $\mathrm{EC}_{\mathrm{NSH}}$ to $\left.\mathrm{EC}_{\mathrm{NSH}}\right)$ plus $\mathrm{OC}_{\mathrm{NSH}}$, (b) relationship of $\mathrm{EC}_{\mathrm{IMP}} \mathrm{TOR}(y$ axis) and $\mathrm{EC}_{\mathrm{NSH}}$ _TOT $\left(x\right.$ axis), and (c) relationship of $\mathrm{EC}_{\mathrm{IMP}}$ TOR $\left(y\right.$ axis) and the sum of $\mathrm{EC}_{\mathrm{NSH}}$ _TOT and OC4 $4_{\mathrm{NSH}}(x$ axis). WODR stands for weighed orthogonal distance regression.

two protocols. The carbon fraction evolved corresponding to the $870^{\circ} \mathrm{C}$ step is classified as OC4 in the NIOSH protocol, while in IMPROVE this fraction is evolved as part of apparent EC (AEC), which is the sum of all the EC fractions before correcting for charred OC. Chow et al. (2001) found NIOSH OC4 can explain most of the EC difference in US samples between the two protocols, and this relationship has been further defined in a PRD study by our group (Eq. 1), where IMPROVE AEC is found to be equivalent to the sum of NIOSH OC4 and NIOSH AEC (Wu et al., 2012).

$\mathrm{AEC}_{\mathrm{IMP}}=\mathrm{AEC}_{\mathrm{NSH}}+\mathrm{OC}_{\mathrm{NSH}}$

HK samples from the current study also confirm this relationship as shown in Fig. 3a (Slope =0.99). It should be noted that, due to the much longer step durations in the IMPROVE protocol, carbon evolved beyond $550^{\circ} \mathrm{C}$ in IMPROVE protocol does not simply map to OC evolved beyond the same temperature point in the NIOSH protocol (i.e., temperature step beyond $550{ }^{\circ} \mathrm{C}$, which includes part of $\mathrm{OC} 3$ and OC4).

The reported IMPROVE TOR EC is the sum of carbon fractions evolved in the $\mathrm{He}-\mathrm{O}_{2}$ stage ( $\mathrm{AEC}_{\mathrm{IMP}}$ ) minus $\mathrm{PC}$ as measured by laser reflectance.

$\mathrm{EC}_{\text {IMP_TOR }}=\mathrm{AEC}_{\mathrm{IMP}}-\mathrm{PC}_{\mathrm{IMP}}$ TOR

Combining Eqs. (1) and (2), the IMPROVE TOR EC can be defined as

$\mathrm{EC}_{\text {IMP_TOR }}=\mathrm{AEC}_{\mathrm{NSH}}+\mathrm{OC}_{\mathrm{NSH}}-\mathrm{PC}_{\mathrm{IMP} \_\mathrm{TOR}}$.

Likewise, the reported NIOSH TOT EC is the sum of carbon fractions evolved in the $\mathrm{He}-\mathrm{O}_{2}$ stage $\left(\mathrm{AEC}_{\mathrm{NSH}}\right)$ minus pyrolysis carbon by laser transmittance.

$\mathrm{EC}_{\text {NSH_TOT }}=\mathrm{AEC}_{\mathrm{NSH}}-\mathrm{PC}_{\text {NSH_TOT }}$

As shown in Fig. 3b, the linear regression slope (2.05) of the scatterplot represents the average discrepancy between $\mathrm{EC}_{\mathrm{IMP}} \mathrm{TOR}\left(y\right.$ axis) and $\mathrm{EC}_{\text {NSH_TOT }}(x$ axis). As embodied in Eqs. (3) and (4), the EC discrepancy can be attributed to two factors: $\mathrm{OC}_{\mathrm{NSH}}$ (thermal effect) and the difference in PC (optical method effect). Thermal effect refers to inter-protocol EC difference caused by temperature step difference. The optical method effect is the interprotocol EC difference introduced by the $\mathrm{PC}$ difference between transmittance and reflectance charring correction. By adding $\mathrm{OC}_{\mathrm{NSH}}$ to the $x$ axis (Fig. $3 \mathrm{c}$ ), the effect of $\mathrm{OC} 4_{\mathrm{NSH}}$ between $y\left(\mathrm{EC}_{\mathrm{IMP}}\right.$ TOR $)$ and $x\left(\mathrm{OC}_{\mathrm{NSH}}+\mathrm{EC}_{\mathrm{NSH} \_\mathrm{TOT}}\right)$ is minimized as embodied in Eqs. (3) and (5), where the slope (1.18) primarily represents the optical method effect caused by the PC difference (PC IMP_TOR Vs. $_{\text {PC }}$ NSH_TOT $_{-}$).

$\mathrm{EC}_{\mathrm{NSH}} \mathrm{TOT}_{-}+\mathrm{OC}_{\mathrm{NSH}}=$

$\mathrm{AEC}_{\mathrm{NSH}}+\mathrm{OC}_{\mathrm{NSH}}-\mathrm{PC}_{\mathrm{NSH}} \mathrm{TOT}_{\mathrm{T}}$

The difference between the slopes in Fig. 3b (slope $=2.05$ ) and Fig. $3 \mathrm{c}$ (slope $=1.18$ ) indicates the contribution of the thermal effect to the EC discrepancy. By examining the relative differences from unity in the two slopes (i.e., 0.18/1.05), it is estimated that $82.86 \%$ of the EC difference by the two protocols in $\mathrm{HK}$ samples is attributed to the thermal effect $\left(\mathrm{OC} 4_{\mathrm{NSH}}\right)$, and the rest $(17.14 \%)$ is due to the PC monitoring, arising from different optical methods used for the charring correction (laser transmittance or reflectance). The reduced $R^{2}$ in Fig. 3b and c compared to Fig. 3a suggest the scatter of data points is due to the optical method effect (PC). The relative contribution of the two factors in the HK samples exhibits a seasonal dependency as shown in Fig. S3. In summer and fall, the optical method effect accounts for $\sim 12 \%$ of the EC discrepancy, while in winter and spring the optical method effect contribution is $35 \%$. This is in part dictated by a lower proportion of $\mathrm{OC}_{\mathrm{NSH}}$ fraction in these two seasons as shown in Fig. S4, leading to an attenuated thermal effect.

It is also found that the optical method effect described above exhibits a dependency on the temperature ramping step. However, PC cannot be compared directly between the two protocols because they evolve under different temperature regimes; thus the PC difference of using the TOR or TOT signal within the protocols is compared as shown in Fig. 4. 

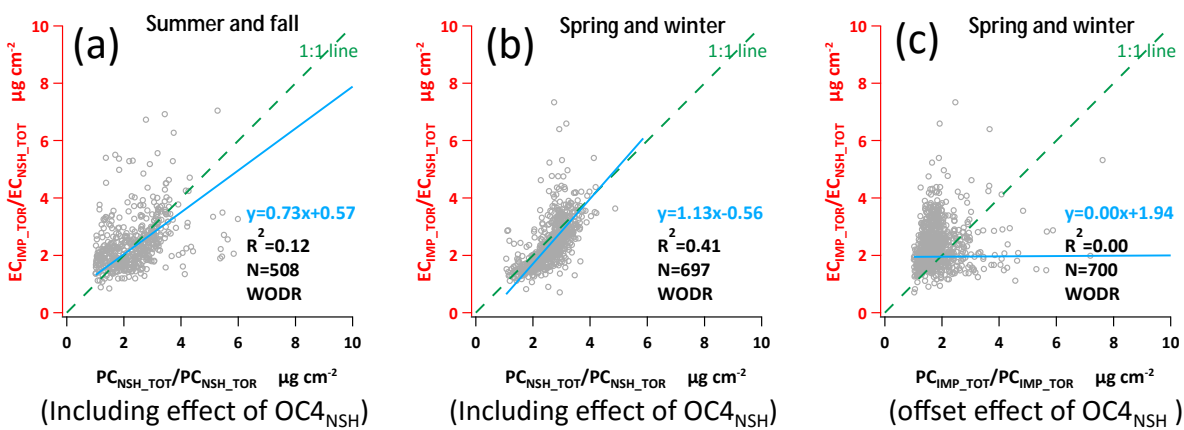

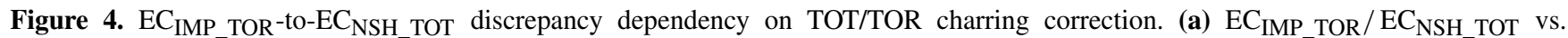

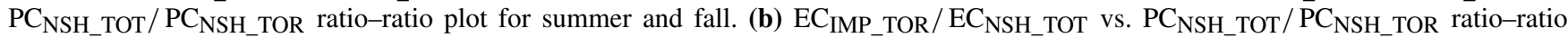

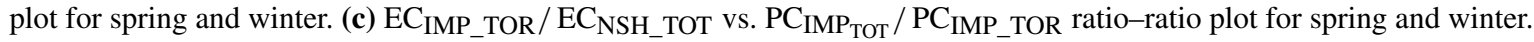

(a) Including effect of
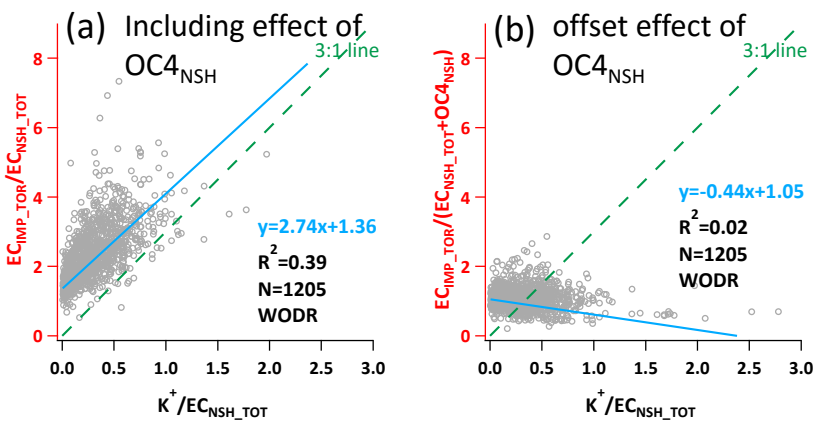

Figure 5. EC IMP $_{\text {TOR }}$-to-EC $\mathrm{NSH}_{\mathrm{NOT}}$ tiscrepancy dependency on $\mathrm{K}^{+} / \mathrm{EC}_{\mathrm{NSH}}$ TOT ratio (biomass burning effect). (a) $\mathrm{EC}_{\mathrm{IMP} \_\mathrm{TOR}} / \mathrm{EC}_{\mathrm{NSH} \text { TOT }}$ vs. $\mathrm{K}^{+} / \mathrm{EC}_{\mathrm{NSH}}$ TOT ratioratio plot. (b) $\mathrm{EC}_{\mathrm{IMP}} \mathrm{TOR} /\left(\mathrm{EC}_{\mathrm{NSH}} \mathrm{TOT}+\mathrm{OC} 4_{\mathrm{NSH}}\right)$ vs. $\mathrm{K}^{+} / \mathrm{EC}_{\mathrm{NSH}}$-TOT ratio-ratio plot.

It is found that the ratio of $\mathrm{EC}_{\mathrm{IMP}}$ TOR $/ \mathrm{EC}_{\text {NSH_tOT }}$ shows a dependency on $\mathrm{PC}_{\text {NSH_tOT }} / \mathrm{PC}_{\text {NSH_TOR }}\left(R^{2}=0.12-0.41\right)$, and the degree of correlation varies by season (Fig. $4 \mathrm{a}$ and $\mathrm{b}$ ). This result agrees well with the higher optical method effect contribution during spring and winter shown in Fig. S3 and discussed above. In contrast, $\mathrm{EC}_{\mathrm{IMP} \_\mathrm{TOR}} / \mathrm{EC}_{\mathrm{NSH} \_\mathrm{TOT}}$ is insensitive to $\mathrm{PC}_{\text {IMP_TOT }} / \mathrm{PC}_{\text {IMP_TOR }}\left(R^{2}=0\right)$ as shown in Fig. 4c. This selective dependency suggests the optical method effect contribution to EC dependency is distinctly sensitive to the degree of charring formed during the $\mathrm{OC} 4_{\mathrm{NSH}}$ stage. Since $\mathrm{PC}_{\mathrm{NSH}}$ contains char formed in the $\mathrm{OC} 4_{\mathrm{NSH}}$ stage while $\mathrm{PC}_{\mathrm{IMP}}$ does not, $\mathrm{OC} 4_{\mathrm{NSH}}$ is the major difference between potential sources of $\mathrm{PC}_{\mathrm{IMP}}$ and $\mathrm{PC}_{\mathrm{NSH}}$ difference.

\subsubsection{Effect of biomass burning on $\mathrm{OC}$ and $\mathrm{EC}$ determination between IMPROVE and NIOSH}

Other potential factors affecting EC discrepancy were also examined. Cheng et al. (2011a) found in Beijing samples that biomass burning can influence the EC discrepancy. Here we use a normalized abundance of $\mathrm{K}^{+}$as an indicator to exam- ine the impact of biomass burning on the EC discrepancy. Figure S5a is the same as Fig. $3 b$ but color coded with the $\mathrm{K}^{+} / \mathrm{EC}_{\mathrm{NSH}}$ TOT ratio to reflect the influence from biomass burning. It reveals a pattern associated with the $\mathrm{EC}_{\mathrm{IMP}_{-} \mathrm{TOR}^{-}}$ to-EC $\mathrm{NSH}_{\mathrm{NS}}$ TOT ratio. To verify this relationship, regressions on the lowest and highest $10 \%$ of $\mathrm{K}^{+} / \mathrm{EC}_{\mathrm{NSH}}$ TOT ratios are shown in Fig. S6b and S6c, respectively. The data from the highest $10 \%$ of $\mathrm{K}^{+} / \mathrm{EC}_{\mathrm{NSH} \_ \text {TOT }}$ ratios have a significantly higher regression slope (slope $=3.19$, Fig. S5c) than the data from the lowest $10 \%$ of $\mathrm{K}^{+} / \mathrm{EC}_{\mathrm{NSH} \text { тOT }}$ ratios (slope $=1.48$, Fig. S5b), implying the EC discrepancy depends on the $\mathrm{K}^{+} / \mathrm{EC}_{\mathrm{NSH}_{-} \text {TOT }}$ ratio. To further distinguish whether the $\mathrm{K}^{+} / \mathrm{EC}_{\text {NSH_TOT }}$ effect is associated with $\mathrm{OC}_{\text {NSH }}$ (thermal effect) or the difference in PC (optical method effect), $\mathrm{OC}_{\mathrm{NSH}}$ is added to the $x$ axis as shown in Fig. S5d-f. By adding $\mathrm{OC}_{\mathrm{NSH}}$ to the $x$ axis, any discrepancy between $y$ and $x$ can be attributed to the optical method effect alone. The slopes of samples from the highest $10 \%$ of $\mathrm{K}^{+} / \mathrm{EC}_{\mathrm{NSH} \_ \text {TOT }}$ ratios $(1.20$, Fig. S5e) and samples from the lowest $10 \%$ of $\mathrm{K}^{+} / \mathrm{EC}_{\mathrm{NSH}}$ TOT ratios $(1.27$, Fig. S5f) are very close to the slope using all samples (1.23, Fig. S5d), implying that the optical method effect is not sensitive to the $\mathrm{K}^{+} / \mathrm{EC}_{\mathrm{NSH}}$ toT ratio. Consequently, the EC discrepancy dependence on the $\mathrm{K}^{+} / \mathrm{EC}_{\mathrm{NSH}}$ TOT ratio is very likely associated with $\mathrm{OC}_{\mathrm{NSH}}$ (thermal effect). Since the intercepts in Fig. S5 are relatively small and their slopes can be represented by ratios, we use ratio-ratio plots to verify the relationship of $\mathrm{K}^{+} / \mathrm{EC}_{\mathrm{NSH}}$ TOT to $\mathrm{OC}_{\mathrm{NSH}}$. As shown in Fig. 5a, when the $\mathrm{K}^{+} / \mathrm{EC}_{\mathrm{NSH}}$ TOT ratio goes up, a larger EC discrepancy is observed; while adding $\mathrm{OC}_{\mathrm{NSH}}$ to the $y$ axis (offsetting the contribution from $\mathrm{OC}_{\mathrm{NSH}}$ ) as shown in Fig. 5b, this relationship no longer holds. The $\mathrm{OC}_{\mathrm{NSH}}$ fraction, as represented by the relative abundance of $\mathrm{OC}_{\mathrm{NSH}}$ in samples $\left(\mathrm{OC} 4_{\mathrm{NSH}} / \mathrm{TC}\right)$, exhibits a dependency on the $\mathrm{K}^{+} / \mathrm{EC}_{\mathrm{NSH}}$ TOT ratio as illustrated in the histograms of Fig. S6. An independent $t$ test (Table S2) was performed, finding the average $\mathrm{OC} 4_{\mathrm{NSH}} / \mathrm{TC}$ ratio of samples from the highest $10 \%$ of $\mathrm{K}^{+} / \mathrm{EC}_{\mathrm{NSH}}$ TOT ratios $(0.27$, Fig. S6c) is significantly higher $(p<0.001)$ than the aver- 

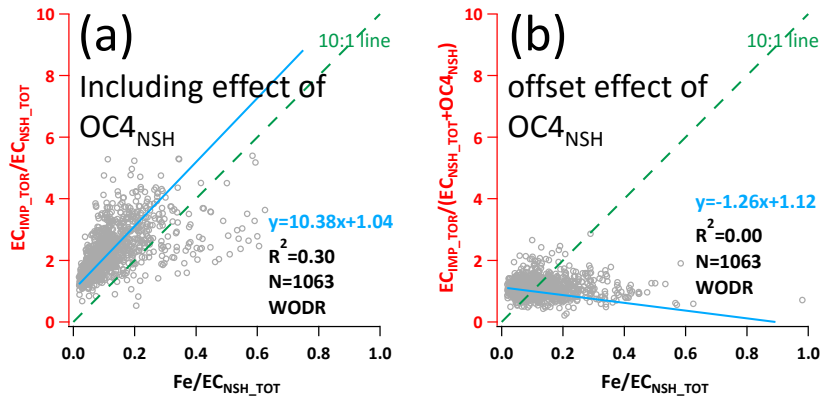

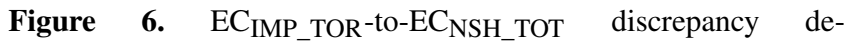
pendency on $\mathrm{Fe} / \mathrm{EC}_{\mathrm{NSH}} \overline{\mathrm{TOT}}_{\mathrm{T}}$ ratio (metal oxide effect). (a) $\mathrm{EC}_{\mathrm{IMP} \_\mathrm{TOR}} / \mathrm{EC}_{\mathrm{NSH} \text { _TOT }} \mathrm{vs}$. Fe / $\mathrm{EC}_{\mathrm{NSH} \text { _TOT }}$ ratio-ratio plot. (b) $\mathrm{EC}_{\mathrm{IMP} \_\mathrm{TOR}} /\left(\mathrm{EC}_{\mathrm{NSH}} \mathrm{TOT}_{\mathrm{T}}+\mathrm{OC}_{\mathrm{NSH}}\right)$ vs. $\mathrm{Fe} / \mathrm{EC}_{\mathrm{NSH}} \mathrm{TOT}$ ratio-ratio plot.

age $\mathrm{OC} 4_{\mathrm{NSH}} / \mathrm{TC}$ ratio of samples from the lowest $10 \%$ of $\mathrm{K}^{+} / \mathrm{EC}_{\mathrm{NSH}}$ TOT ratios, which reveals that the $\mathrm{OC}_{\mathrm{NSH}}$ fraction and $\mathrm{K}^{+} / \mathrm{EC}_{\mathrm{NSH} \_ \text {TOT }}$ ratio are positively correlated. As discussed before, the $\mathrm{OC} 4_{\mathrm{NSH}}$ fraction can affect the $\mathrm{EC}$ discrepancy, which is the reason that biomass burning can influence the EC discrepancy.

\subsubsection{Effect of metal oxides on $\mathrm{OC}$ and $\mathrm{EC}$ determination between IMPROVE and NIOSH}

A suite of laboratory studies have revealed the presence of metal oxides in aerosol samples can alter the EC / OC ratio, by either lowing the EC oxidation temperature or enhancing OC charring (Murphy et al., 1981; Wang et al., 2010; Bladt et al., 2014). As a result, the distribution of carbon fractions is impacted during the analysis, affecting the inter-protocol EC discrepancy. As shown in Figs. 6a and S7, the EC discrepancy positively correlates with normalized $\mathrm{Fe}$ abundance ( $\mathrm{Fe} / \mathrm{EC}_{\mathrm{NSH}}$ TOT ratio), suggesting that a higher fraction of metal oxide can increase the EC divergence across the two protocols. If $\mathrm{OC} 4_{\mathrm{NSH}}$ is added to cancel out the discrepancy contribution from the thermal effect (Figs. 6b and S7), the discrepancy due to the optical method effect alone shows no dependency on Fe abundance. Similar dependency is also found in other metal oxides like $\mathrm{Al}$ as shown in Fig. S8. These results imply that metal-oxide-induced EC divergence is mainly associated with the $\mathrm{OC} 4_{\mathrm{NSH}}$ fraction.

\subsection{Comparison of IMPROVE TOR EC reconstruction approaches for Hong Kong samples}

\subsubsection{Description of two reconstruction methods}

It is of great interest to determine the best estimation for $\mathrm{EC}_{\text {IMP_TOR }}$ when only NIOSH TOT analysis is available. This study provides an opportunity to examine different empirical reconstruction approaches for $\mathrm{EC}_{\text {IMP_TOR }}$ using the $\mathrm{EC}_{\mathrm{NSH}}$ _TOT data. In total, four approaches are investigated; two of them are discussed below, and the other two are discussed in the Supplement. The first method is direct regression (M1), which applies the relationship obtained from Fig. S9 to reconstruct EC $\mathrm{CIMP}_{\text {ITOR: }}$

M1 : $\mathrm{EC}_{\text {IMP_TOR }}=a \times \mathrm{EC}_{\text {NSH_TOT }}+b$

Then, reconstructed $\mathrm{OC}_{\mathrm{IMP}} \mathrm{TOR}$ can be obtained by subtracting reconstructed $\mathrm{EC}_{\mathrm{IMP} \_\mathrm{TOR}}$ from $\mathrm{TC}_{\mathrm{NSH}}$ :

reconstructed $\mathrm{OC}_{\text {IMP_TOR }}=$

$\mathrm{TC}_{\mathrm{NSH}}$ - reconstructed $\mathrm{EC}_{\mathrm{IMP}} \mathrm{TOR}$.

Further reconstruction methods may deconstruct $\mathrm{EC}_{\text {IMP_TOR }}$ into several terms based on analysis principles and apply regression only on the unknown terms. Since only a partial regression is involved, theoretically, this approach can provide more accurate reconstruction results. Relationships found in Sect. 3.2 can also be used to refine the reconstruction.

The second approach (M2) employs partial regression. In Eq. (3), $\mathrm{PC}_{\text {IMP_TOR }}$ is the only unknown term on the right-

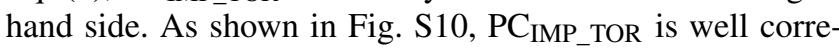
lated with $\mathrm{PC}_{\mathrm{NSH}} \mathrm{TOR}$, which is known from NIOSH analysis. Therefore, Eq. (3) can be approximated as

M2: EC $_{\text {IMP_TOR }}=$

$\mathrm{AEC}_{\mathrm{NSH}}+\mathrm{OC}_{\mathrm{NSH}}-\left(a \times \mathrm{PC}_{\mathrm{NSH}}{ }_{-\mathrm{TOR}}+b\right)$.

M2 can be further improved if chemical composition data are available. As discussed above, the abundance of $\mathrm{K}^{+}$ and $\mathrm{Fe}$ can affect EC discrepancy. To reflect the contributions from these factors, $\mathrm{PC}_{\mathrm{NSH}_{-} \mathrm{TOR}}, \mathrm{K}^{+}$, and $\mathrm{Fe}$ are included to approximate $\mathrm{PC}_{\mathrm{IMP}}$ TOR by multiple linear regression (MLR), then Eq. (3) can be rewritten as

$$
\begin{aligned}
\text { M2 }-1 & : \mathrm{EC}_{\text {IMP_TOR }}=\mathrm{AEC}_{\mathrm{NSH}}+\mathrm{OC}_{\mathrm{NSH}} \\
& -\left(a_{1} \times \mathrm{PC}_{\mathrm{NSH} \_\mathrm{TOR}}+a_{2} \times \mathrm{K}^{+}+a_{3} \times \mathrm{Fe}+b\right) .
\end{aligned}
$$

$a_{1}, a_{2}$, and $a_{3}$ are MLR coefficients. $\mathrm{K}^{+}$is measured by ion chromatography, and $\mathrm{Fe}$ is detected by $\mathrm{X}$-ray fluorescence.

An alternative reconstruction method (M3) is discussed in the Supplement. In brief, M3 is based on the linear relationship between ( $\left.\mathrm{PC}_{\text {NSH_TOT }}-\mathrm{PC}_{\mathrm{NSH}}{ }_{-} \mathrm{TOR}\right)$ and $\left(\mathrm{PC}_{\mathrm{NSH}} \mathrm{TOT}^{-}\right.$ $\mathrm{PC}_{\text {IMP_TOR }}$ ) for reconstruction.

\subsubsection{Reconstruction of $2013 \mathrm{OC}$ and EC using parameters from 2011-2012 data}

In this section, blind tests are performed to compare the performance of the two reconstruction methods (M1 and M2). Results of M2 - 1 and M3 reconstruction approaches are discussed in the Supplement. Data from 2011-2012 are used to obtain the necessary parameters ( $\mathrm{a}$ and b) for M1 and M2 as shown in Eqs. (6) and (8), respectively. Since these parameters may vary temporally and spatially, two scenarios 
Table 2. Regression parameters for OC and EC reconstruction equations.

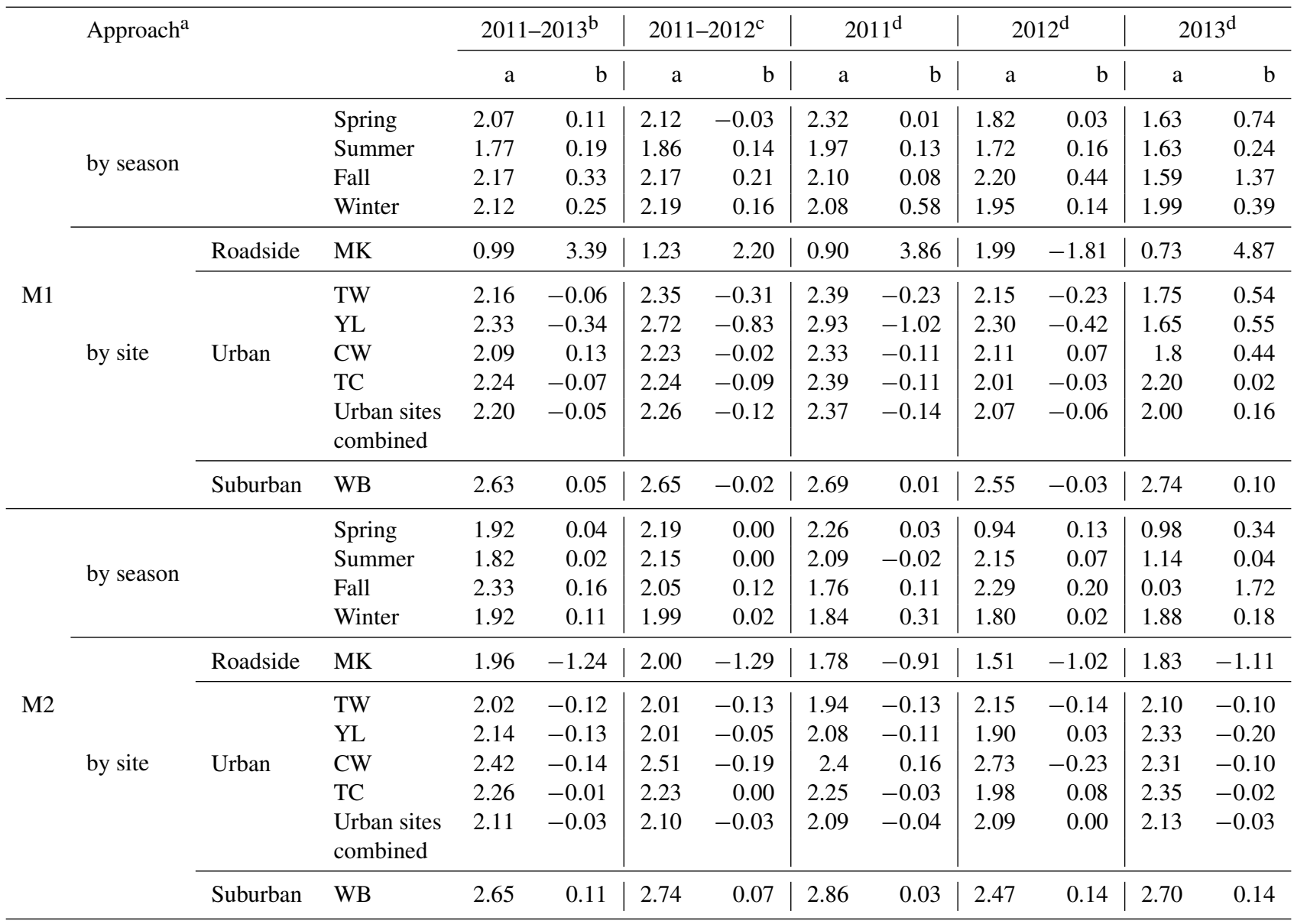

\footnotetext{
a The two reconstruction method equations are M1 : $\mathrm{EC}_{\mathrm{IMP} \_\mathrm{TOR}}=a \times \mathrm{EC}_{\mathrm{NSH} \_\mathrm{TOT}}+b ; \mathbf{M} 2: \mathrm{EC}_{\mathrm{IMP} \_\mathrm{TOR}}=\mathrm{AEC}_{\mathrm{NSH}}+\mathrm{OC}_{\mathrm{NSH}}-\left(a \times \mathrm{PC}_{\mathrm{NSH}} \mathrm{TOR}+b\right)$.

${ }^{b}$ Regression parameters are derived from 2011-2013 data. ${ }^{\mathrm{c}}$ Regression parameters are derived from 2011-2012 data. ${ }^{\mathrm{d}}$ Regression parameters are derived from a single year's data.
}

are considered for parameterization: scenario 1, seasonal specific parameters for each season with samples from all sites; scenario 2, site-specific parameters for all samples from a site or combined sites with a similar site characteristic. Detailed parameters are summarized in Table 2. These parameters are then applied to NIOSH data in 2013, and reconstructed $\mathrm{EC}_{\text {IMP_TOR }}$ and $\mathrm{OC}_{\text {IMP_TOR }}$ concentrations are calculated and compared with measured $2013 \mathrm{EC}_{\mathrm{IMP}} \mathrm{TOR}$ and $\mathrm{OC}_{\mathrm{IMP}}$ TOR to evaluate the performance of OC and EC reconstruction by the two scenarios. Since two scenarios are considered in each reconstruction method, there are four combinations of reconstruction results for M1 and M2.

Reconstructed EC by M1 is compared with measured EC in Fig. 7a and b. The $R^{2}$ of the season-specific (Fig. 7a) and site-specific reconstruction (Fig. 7b) are comparable with each other. Reconstructed EC is also compared with measured EC using histograms as shown in Fig. S15. The mean concentration by site-specific reconstruction agrees better than the season-specific reconstruction. The frequency distribution of the relative difference of reconstructed vs. mea- sured EC exhibits a similar distribution between the seasonand site-specific reconstructions (Fig. S16). OC reconstruction by M1 is shown in Fig. 8a and b, revealing reconstruction by site-specific parameters can increase the $R^{2}$, with a tradeoff of higher average bias (slope $=1.14$ ). The seasonal or site-specific parameters yield similar reconstructed OC distributions as shown in Figs. S17 and S18. The OC / EC ratios reconstructed by $\mathrm{M} 1$ are overestimated by a factor of 2 as shown by the slopes in Fig. 9. The reconstructed $\mathrm{OC} / \mathrm{EC}$ distribution is significantly broader than the measured OC / EC ratios as shown in Figs. S19 and S20. This is an expected result of reconstructed $\mathrm{OC}$ and $\mathrm{EC}$ inherently having bias of opposite signs (i.e., if reconstructed OC is biased higher, then reconstructed EC would be biased lower), amplifying the bias in the ratio quantity.

Results of $\mathrm{EC}_{\mathrm{IMP}}$ TOR reconstruction by $\mathrm{M} 2$ are shown in Fig. 7c and d. Slopes by M2 are the closest to the unity of all the methods, implying that M2 can provide better accuracy than M1. M2 reconstruction by site exhibits the highest $R^{2}$ among all reconstruction scenarios. The supe- 

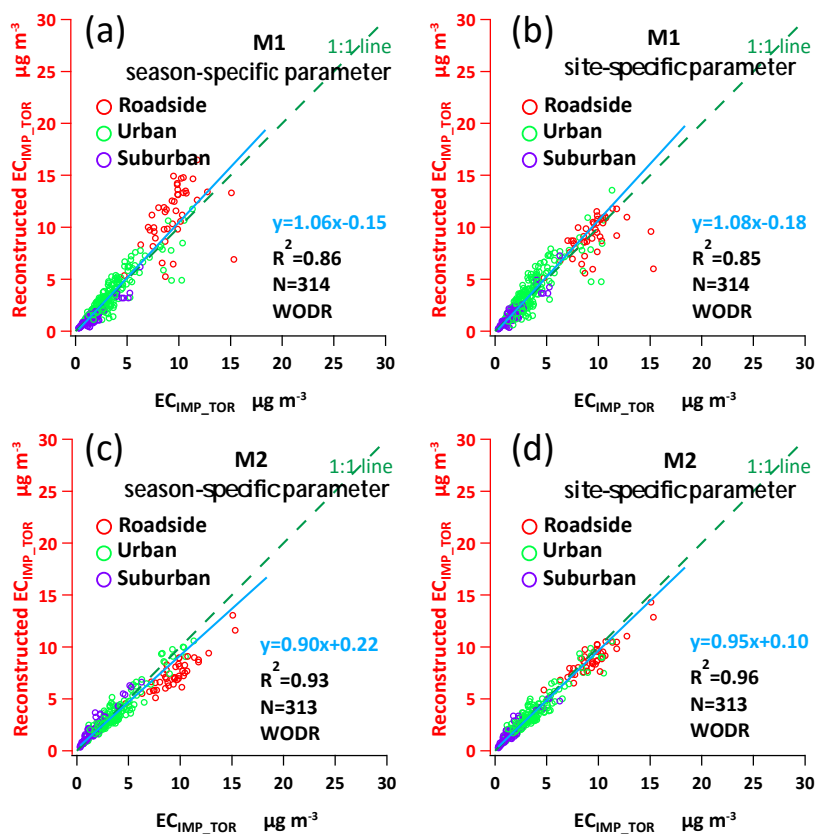

Figure 7. Comparison of reconstructed $\mathrm{EC}_{\mathrm{IMP}} \mathrm{TOR}$ and measurement $\mathrm{EC}_{\mathrm{IMP}} \mathrm{TOR}$ in the year 2013. (a) Regression by seasonspecific parameters using M1. (b) Regression by site-specific parameters using M1. (c) Regression by season-specific parameters using M2. (d) Regression by site-specific parameters using M2.

rior performance of $\mathrm{M} 2$ by site-specific parameters is also evidenced by the sharpened distribution peak around zero for the relative difference of measured and reconstructed EC (Fig. S16d). OC reconstruction by M2 using site-specific parameters (Fig. 8d) yields a higher $R^{2}$ than the season-specific scenario (Fig. 8c). The OC relative difference distribution is sharpest in the site-specific-parameters scenario as shown in Fig. S18d. The OC / EC ratios reconstructed by M2 are underestimated by 22 to $72 \%$ as shown in Fig. 9 , with a low $R^{2}$ ranging from 0.3 to 0.46 . The OC / EC bias is also evidenced by significantly different histograms between the distinctly sharper peak of the reconstructed OC / EC compared with measured OC / EC (Fig. S19c and d).

From the comparisons shown above, it is obvious that the M2 site-specific-parameters scenario can provide the best performance in OC and EC reconstruction, evidenced by regression slopes being closest to unity and the sharpest frequency distribution histograms of OC or EC differences between reconstructed and measured values. However, the OC / EC ratio is not well reproduced by the two methods; it is overestimated and underestimated by M1 and M2, respectively.

To investigate the stability of various parameters used in the two reconstruction scenarios, we also calculate reconstruction parameters for individual years from 2011 to 2013 as well as for the entire 3-year dataset as listed in Table 2. The reconstruction parameters are of similar values between

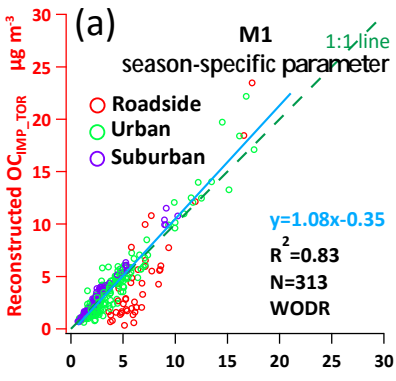

$$
\text { OC } \mathrm{CMP}_{-} \text {TOR } \quad \mu \mathrm{g} \mathrm{\textrm {m } ^ { - 3 }}
$$
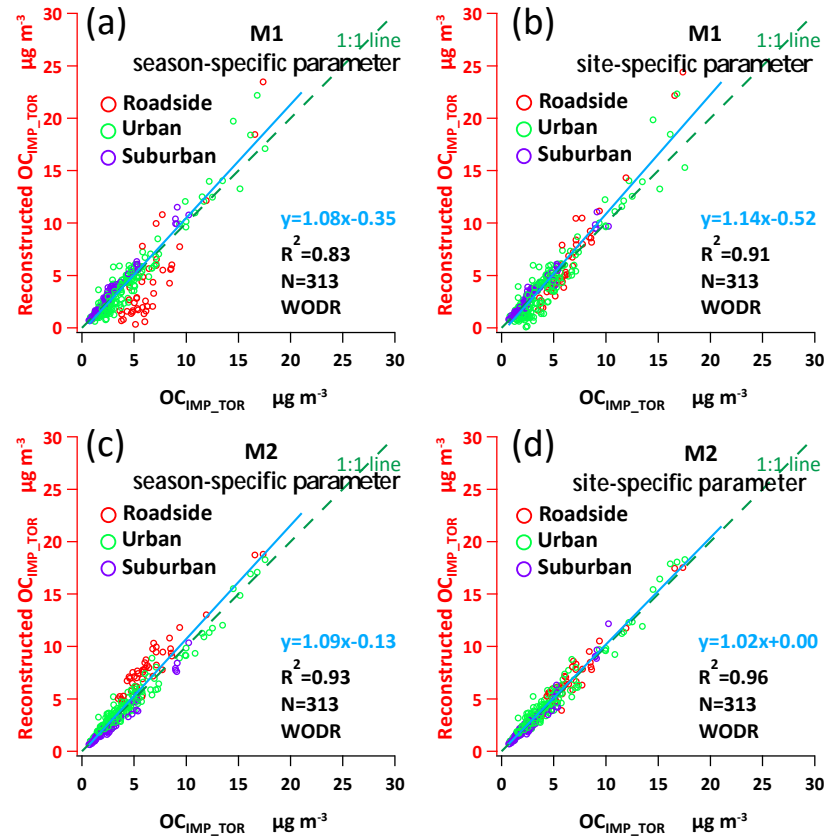

Figure 8. Reconstruction of $\mathrm{OC}_{\mathrm{IMP}}{ }_{\mathrm{C}} \mathrm{TOR}$ calculated using Eq. (7). (a) Reconstruction by season-specific parameters using M1. (b) Reconstruction by site-specific parameters using M1. (c) Reconstruction by season-specific parameters using M2. (d) Reconstruction by site-specific parameters using M2.
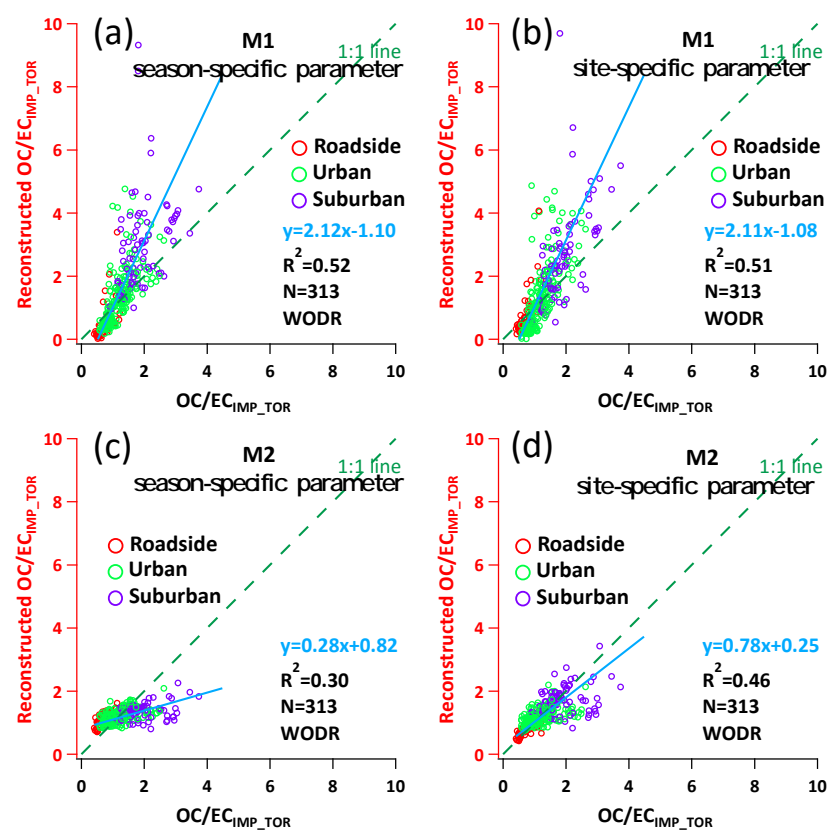

Figure 9. Reconstruction results of OC / EC IMP_TOR - (a) Reconstruction by season-specific parameters using M1. (b) Reconstruction by site-specific parameters using M1. (c) Reconstruction by season-specific parameters using M2. (d) Reconstruction by sitespecific parameters using M2. 

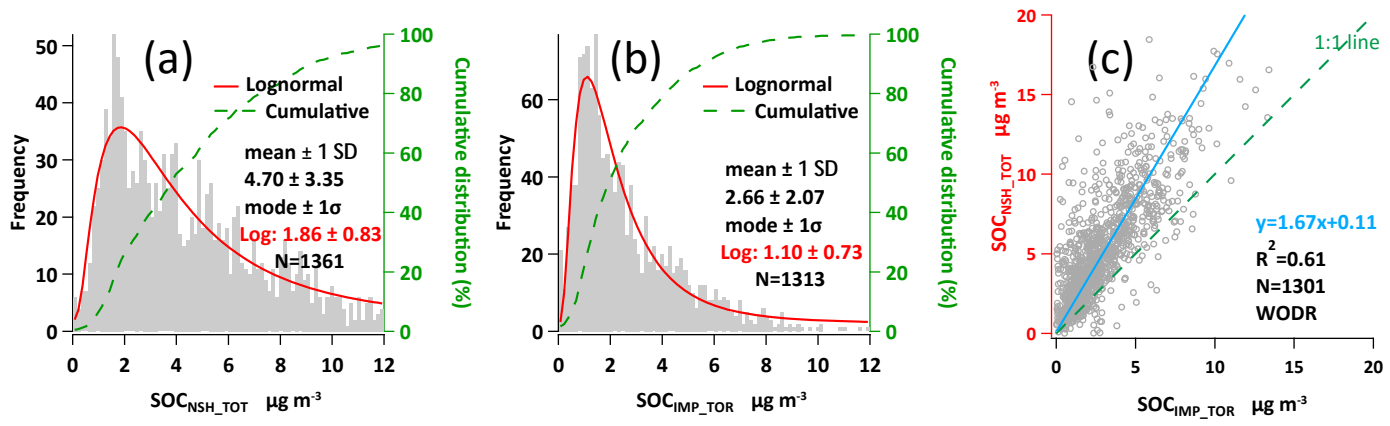

Figure 10. Comparison of SOC by NIOSH and IMPROVE. (a) SOC estimation from NIOSH TOT data. (b) SOC estimation from IMPROVE TOR data. (c) The relationship between $\mathrm{SOC}_{\mathrm{NSH} \_\mathrm{TOT}}$ and $\mathrm{SOC}_{\mathrm{IMP}}$ TOR.
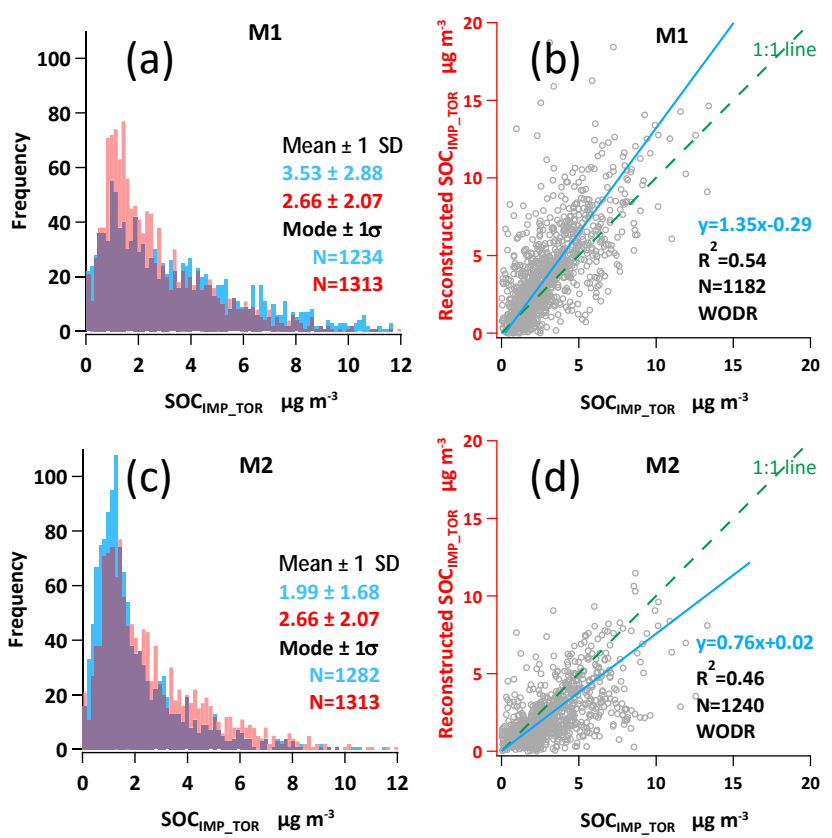

Figure 11. Histogram comparison of original $\mathrm{SOC}_{\mathrm{IMP}} \mathrm{TOR}$ (in red) with SOC IMP_TOR (in blue) reconstructed by M1 (a) and by M2 (c). Scatterplot comparison of original SOC $\mathrm{IMP}_{-} \mathrm{TOR}$ (in $x$ axis) with

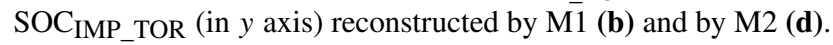

years, implying these methods are robust for future reconstruction applications. The implementation of M1 to all urban site data (without site or seasonal specificity) yields the following equation, and this equation is recommended for urban site data conversion.

M1 (urban data) : $\mathrm{EC}_{\mathrm{IMP}}$ TOR $=$

$2.20 \times \mathrm{EC}_{\text {NSH_TOT }}-0.05$

For a heavily trafficked roadside environment, the recommended slope and intercept are 0.99 and 3.39, respectively. For suburban environments with light EC loadings, the recommended values are 2.63 for slope and -0.05 for intercept.
The M2 site-specific parameters exhibit weaker site dependence than the M1 method, making M2 more suitable for expanding its application in other regions. As a result, M2 site-specific parameters obtained from the 3-year dataset are recommended for future reconstruction applications in Hong Kong (Table 2). The equation for urban environments is shown below:

M2(urban data) : $\mathrm{EC}_{\mathrm{IMP} \_\mathrm{TOR}}=$

$\mathrm{AEC}_{\mathrm{NSH}}+\mathrm{OC}_{\mathrm{NSH}}-\left(2.11 \times \mathrm{PC}_{\mathrm{NSH}} \_\mathrm{TOR}-0.03\right)$.

We note that the $\mathrm{AEC}_{\mathrm{NSH}}, \mathrm{OC}_{\mathrm{NSH}}$, and $\mathrm{PC}_{\mathrm{NSH}} \mathrm{TOR}$ inputs required in $\mathrm{M} 2$ are not always available for data users, as they are typically not reported by analysis laboratories.

Similarly, M2 - 1 site-specific parameters obtained from the 3-year dataset are recommended for future reconstruction applications in Hong Kong if $\mathrm{K}^{+}$and $\mathrm{Fe}$ data are available (Table S4). The equation for urban environments is given below:

M2 -1 (urban data) : $\mathrm{EC}_{\mathrm{IMP}} \mathrm{TOR}_{\mathrm{TO}}=$

$\mathrm{AEC}_{\mathrm{NSH}}+\mathrm{OC}_{\mathrm{NSH}}-\left(0.94 \times \mathrm{PC}_{\mathrm{NSH}} \_\mathrm{TOR}+1.60\right.$

$\left.\times \mathrm{K}^{+}+1.47 \times \mathrm{Fe}+0.00\right)$.

Monthly variations of measured and reconstructed IMPROVE TOR EC and OC (M2, site-specific parameters) are shown in Fig. S21, clearly showing the reconstructed OC and EC data can reproduce the monthly trend quite well as compared with the measured data. This demonstrates that the reconstruction equations can provide a means to establish temporal trends for OCEC data produced using different analysis protocols.

\subsection{Implications for secondary OC (SOC) estimation}

The EC tracer method (Turpin and Huntzicker, 1995) is a widely used approach for SOC estimation since it only requires measured $\mathrm{OC}$ and $\mathrm{EC}$ as input:

$\mathrm{SOC}=\mathrm{OC}_{\text {total }}-\left(\frac{\mathrm{OC}}{\mathrm{EC}}\right)_{\text {pri }} \times \mathrm{EC}-\mathrm{OC}_{\text {non-comb }}$, 
where $(\mathrm{OC} / \mathrm{EC})_{\text {pri }}$ is the $\mathrm{OC} / \mathrm{EC}$ ratio in freshly emitted combustion aerosols, $\mathrm{OC}_{\text {total }}$ and $\mathrm{EC}$ are from the measurements, and $\mathrm{OC}_{\text {non-comb }}$ is the $\mathrm{OC}$ fraction from noncombustion sources (i.e., biogenic emissions). Since the $\mathrm{OC}_{\text {non-comb }}$ is usually small, it is considered as zero to simplify the calculation in our study. The key to the EC tracer method is to estimate a proper (OC/EC) pri. Our previous study proved that the minimum R squared method (MRS) is more accurate than the conventional subset percentile or minimum OC / EC ratio approaches (Wu and Yu, 2016). Therefore, MRS is employed for SOC calculation in this study. In this section, two aspects are discussed regarding SOC estimation: (1) variability of OC and EC by different protocols and the impacts on SOC estimation and (2) the usability of reconstructed $\mathrm{EC}_{\mathrm{IMP} \_ \text {TOR }}$ and $\mathrm{OC}_{\mathrm{IMP}}$ TOR for SOC estimation.

Since the proportion of different primary emission sources is expected to vary by season, $(\mathrm{OC} / \mathrm{EC})_{\mathrm{pri}}$ is calculated by MRS for each season (Table S3) using all 3 years of data (2011-2013). As shown in Fig. 10, SOC by NIOSH TOT (mean concentration: $4.70 \mu \mathrm{g} \mathrm{m}^{-3}$ ) is higher than by the IMPROVE TOR protocol (mean concentration: $2.66 \mu \mathrm{g} \mathrm{m}^{-3}$ ). On average, $\mathrm{SOC}_{\text {NSH_toT }}$ is 1.67 times higher than SOC $_{\text {IMP }}$ TOR as suggested by the regression slope in Fig. 10c. Although the absolute SOC concentrations by the two protocols are quite divergent, the $R^{2}(0.61)$ suggests that the two SOCs are moderately correlated. WSOC has been recognized as a good indicator of SOC formation (Sullivan et al., 2004), but WSOC contribution from primary emission is not negligible (Graham et al., 2002). Instead of using WSOC directly, we use secondary WSOC (SWSOC) as an indicator to verify the SOC results. SWSOC can be calculated from the following equation:

SWSOC $=$ WSOC - sugars $\times\left(\frac{\text { WSOC }}{\text { sugars }}\right)_{\text {pri }}$.

In Eq. (14), sugars - which include levoglucosan, mannosan, and galactosan - are used as a tracer to derive SWSOC based on the primary ratio (5.28, Fig. S22) obtained from a biomass burning source profile measured in the PRD region (Lin et al., 2010). The relationship between SWSOC and SOC is examined in Fig. S23 for the WB site where sugars and WSOC data are available. SWSOC accounts for $61 \%$ of $\mathrm{SOC}_{\text {NSH_tOT }}$, which is comparable with the WSOC / $\mathrm{SOC}_{\text {NSH_TOT }}$ ratio observed in Beijing (50$70 \%$ ) by Cheng et al. (2011b). The SWSOC-to-SOC IMP_TOR regression slope is close to unity (0.92), implying that SOC by IMPROVE TOR could be underestimated. SOC by both SOC $_{\text {NSH_TOT }}$ and SOC IMP_TOR is well correlated with SWSOC, confirming the significant contribution of WSOC to $\mathrm{SOC}$ in this region. $\mathrm{SOC}_{\mathrm{NSH}}$ TOT exhibits a higher correlation $\left(R^{2}=0.92\right)$ with WSOC than SOC IMP_TOR $_{\text {IM }}\left(R^{2}=0.86\right)$, which is in good agreement with the study in Beijing (Cheng et al., 2011b), suggesting that NIOSH TOT might be more reasonable for SOC estimation.
The usability of reconstructed $\mathrm{EC}_{\mathrm{IMP}}$ TOR and $\mathrm{OC}_{\mathrm{IMP}} \mathrm{TOR}$ (using M1 and M2) for SOC estimation is investigated. Results of M2 - 1 and M3 are discussed in Supplement. To account for the temporal variations of $(\mathrm{OC} / \mathrm{EC})_{\mathrm{pri}}$, seasonal $(\mathrm{OC} / \mathrm{EC})_{\text {pri }}$ values are calculated using $\mathrm{OC}$ and $\mathrm{EC}$ reconstructed by M1 and M2 (Table S3). These (OC / EC) pri values are then subject to SOC estimation following Eq. (13). It is very clear that the frequency distribution of reconstructed SOCs deviates from the SOC derived from measured OC and EC (Fig. 11). The SOC by M1 is higher than the original SOC, evidenced by average concentrations (3.53 vs. $2.66 \mu \mathrm{g} \mathrm{m}^{-3}$ ) and also confirmed by the regression slope (1.35). On the other hand, SOC by M2 is underestimated by $30-40 \%$. The moderate $R^{2}$ (Fig. 11d) also suggests the SOC by reconstructed $\mathrm{EC}_{\mathrm{IMP}}$ TOR and $\mathrm{OC}_{\mathrm{IMP}}$ _TOR is poorly correlated with SOC by measured $\mathrm{EC}_{\mathrm{IMP}} \mathrm{P}_{-} \mathrm{TOR}$ and $\mathrm{OC}_{\mathrm{IMP}} \mathrm{TOR}$. The significant bias and moderate correlations suggest that reconstructed $\mathrm{EC}_{\mathrm{IMP}} \mathrm{TOR}_{\mathrm{T}}$ and $\mathrm{OC}_{\mathrm{IMP}} \mathrm{IOR}_{\mathrm{T}}$ are not suitable for SOC estimation.

\section{Conclusions}

In this study, we use a large dataset that has good temporal (3 years) and spatial coverage (roadside, urban, rural) in Hong Kong to investigate the OC and EC determination discrepancy between NIOSH TOT and IMPROVE TOR protocols. NIOSH TOT reported lower EC (higher OC) than IMPROVE TOR. The divergence between the two protocols is attributed to two effects: thermal effect and optical method effect. The thermal effect is due to the higher PIMT in NIOSH $\left(870^{\circ} \mathrm{C}\right)$ than IMPROVE $\left(550^{\circ} \mathrm{C}\right)$ and the allocation of the $\mathrm{OC}_{\mathrm{NSH}}$ fraction. The optical method effect is a result of different laser signals used by the two protocols (laser transmittance by NIOSH vs. laser reflectance by IMPROVE).

The equivalence between $\mathrm{AEC}_{\mathrm{IMP}}$ and sum of $\mathrm{OC}_{\mathrm{NSH}}$ and $\mathrm{AEC}_{\mathrm{NSH}}$ is confirmed in the current study, and by offsetting the discrepancy from the thermal effect $\left(\mathrm{OC}_{\mathrm{NSH}}\right)$, the contribution from laser correction can be quantified. It is found that on average the thermal effect accounted for $83 \%$ of the EC disagreement, while $17 \%$ is attributed to the optical method effect. The contribution of the two effects exhibits a clear seasonal dependency, with a more pronounced optical method effect in spring and winter $(\sim 35 \%)$.

The intensity of biomass burning influence can affect EC divergence between the two protocols. Samples influenced by biomass burning (evidenced by higher $\mathrm{K}^{+} / \mathrm{EC}_{\mathrm{NSH}}$ тот ratio) come with higher $\mathrm{OC} 4_{\mathrm{NSH}}$ abundance (higher $\mathrm{OC}_{\mathrm{NSH}} / \mathrm{TC}$ ratio), leading to larger EC divergence between the two protocols. The abundance of metal oxide in samples can also affect EC discrepancy, with a larger EC difference observed when a higher fraction of metal oxide is present in the ambient samples.

Four IMPROVE TOR EC reconstruction approaches (M1, M2, M2 - 1, and M3) are proposed. For each approach, two 
parameterization scenarios are considered, including seasonspecific parameters and site-specific parameters. The implementation of M1 to all urban sites (without considering seasonal specificity) yields the following equation:

$\mathrm{M1}($ urban data $): \mathrm{EC}_{\mathrm{IMP} \_\mathrm{TOR}}=2.20 \times \mathrm{EC}_{\mathrm{NSH} \_\mathrm{TOT}}-0.05$.

Considering site specificity yields slightly better reconstruction performance, with the site-specific slope value varying from 2.16 to 2.33 for the urban sites. The suburban site produces a higher slope value (2.63), while the roadside (MK) data produce a noticeably lower slope value (0.99). Hence, roadside samples (i.e., typically significant EC loadings) need to be processed separately and applied its own site-specific parameters for reconstruction when using the M1 equation. The comparisons show that M2 - 1 with sitespecific parameters provides the best reconstruction results, and the regression parameters are given in Tables 2 and S4.

SOC estimation using OC and EC by the two protocols is compared. Based on the SWSOC-to-SOC ratio and correlation coefficients, it is found that SOC concentrations derived from NIOSH TOT are likely more reasonable than those from IMPROVE TOR. The reconstructed $\mathrm{EC}_{\mathrm{IMP}}$ TOR and $\mathrm{OC}_{\mathrm{IMP}}$ TOR for SOC estimation are shown to be unsuitable due to the poor reconstruction of the OC / EC ratio.

\section{Recommendations for applying OCEC reconstruction}

It should be noted that the conversion equations established in this work are based on the fact that all OCEC data analysis was done by the same analyzer. Other instrument-specific parameters might influence the regression if multiple instruments are used in obtaining the OCEC data. For example, temperature offset has been found to vary by instrument in different labs (Panteliadis et al., 2015). Oven soiling and aging have also been found to have an optical influence that introduces uncertainties in the results (Chiappini et al., 2014).

Four reconstruction approaches are proposed in this study; the selection of which to use depends on the degree of availability of the dataset. If the dataset has only OC and EC concentrations and no detailed carbon fraction information, M1 is preferred. If the dataset also comes with carbon fraction information, M2 and M3 are suggested. If chemical speciation data are available, such as $\mathrm{K}^{+}$and $\mathrm{Fe}, \mathrm{M} 2-1$ is recommended to minimize the effect from biomass burning and metal oxides.

\section{Data availability}

OC, EC, inorganic ions and elements data used in this study are available from Hong Kong Environmental Protection Department (enquiry@epd.gov.hk).

\section{The Supplement related to this article is available online at doi:10.5194/amt-9-4547-2016-supplement.}

Acknowledgements. This project was partially supported by the Hong Kong Environment Protection Department (HKEPD) (AS 10-231, 11-03973, and 12-04384). We thank HKEPD for making available the data for this work. We are indebted to Peter Louie for his relentless efforts in pushing for the best possible $\mathrm{PM}_{2.5}$ speciation measurements in Hong Kong.

Edited by: W. Maenhaut

Reviewed by: two anonymous referees

\section{References}

Birch, M. E. and Cary, R. A.: Elemental carbon-based method for monitoring occupational exposures to particulate diesel exhaust, Aerosol. Sci. Technol., 25, 221-241, 1996.

Bladt, H., Ivleva, N. P., and Niessner, R.: Internally mixed multicomponent soot: Impact of different salts on soot structure and thermo-chemical properties, J. Aerosol. Sci., 70, 26-35, doi:10.1016/j.jaerosci.2013.11.007, 2014.

Boggs, P. T., Donaldson, J. R., and Schnabel, R. B.: Algorithm 676: ODRPACK: software for weighted orthogonal distance regression, ACM T. Math. Software., 15, 348-364, 1989.

Bond, T. C., Zarzycki, C., Flanner, M. G., and Koch, D. M.: Quantifying immediate radiative forcing by black carbon and organic matter with the Specific Forcing Pulse, Atmos. Chem. Phys., 11, 1505-1525, doi:10.5194/acp-11-1505-2011, 2011.

Cheng, Y., Duan, F.-K., He, K.-B., Zheng, M., Du, Z.-Y., Ma, Y.L., and Tan, J.-H.: Intercomparison of thermal-optical methods for the determination of organic and elemental carbon: influences of aerosol composition and implications, Environ. Sci. Technol., 45, 10117-10123, doi:10.1021/es202649g, 2011a.

Cheng, Y., He, K.-B., Duan, F.-K., Zheng, M., Du, Z.-Y., Ma, Y.-L., and Tan, J.-H.: Ambient organic carbon to elemental carbon ratios: Influences of the measurement methods and implications, Atmos. Environ., 45, 2060-2066, doi:10.1016/j.atmosenv.2011.01.064, 2011b.

Cheng, Y., He, K.-B., Duan, F.-K., Du, Z.-Y., Zheng, M., and Ma, Y.-L.: Ambient organic carbon to elemental carbon ratios: Influence of the thermal-optical temperature protocol and implications, Sci. Total Environ., 468-469, 1103-1111, doi:10.1016/j.scitotenv.2013.08.084, 2014.

Chiappini, L., Verlhac, S., Aujay, R., Maenhaut, W., Putaud, J. P., Sciare, J., Jaffrezo, J. L., Liousse, C., Galy-Lacaux, C., Alleman, L. Y., Panteliadis, P., Leoz, E., and Favez, O.: Clues for a standardised thermal-optical protocol for the assessment of organic and elemental carbon within ambient air particulate matter, Atmos. Meas. Tech., 7, 1649-1661, doi:10.5194/amt-7-1649-2014, 2014.

Chow, J. C., Watson, J. G., Pritchett, L. C., Pierson, W. R., Frazier, C. A., and Purcell, R. G.: The DRI thermal optical reflectance carbon analysis system - description, evaluation and applications in United-States air-quality studies, Atmos. Environ., 27, 11851201, 1993. 
Chow, J. C., Watson, J. G., Crow, D., Lowenthal, D. H., and Merrifield, T.: Comparison of IMPROVE and NIOSH carbon measurements, Aerosol. Sci. Technol., 34, 23-34, doi:10.1080/027868201300081923, 2001.

Chow, J. C., Watson, J. G., Kohl, S., Gonzi, M., Chen, L.-W. A., and Chai, W.: Measurements and validation for the twelve month particulate matter study in Hong Kong, Hong Kong Environmental Protection Department, Hong Kong, 2002.

Chow, J. C., Watson, J. G., Chen, L. W. A., Arnott, W. P., and Moosmuller, H.: Equivalence of elemental carbon by thermal/optical reflectance and transmittance with different temperature protocols, Environ. Sci. Technol., 38, 4414-4422, doi:10.1021/Es034936u, 2004.

Chow, J. C., Watson, J. G., Kohl, S., Gonzi, M., Chen, L.-W. A., and Chai, W.: Measurements and validation for the twelve month particulate matter study in Hong Kong, Hong Kong Environmental Protection Department, Hong Kong, 2006.

Chow, J. C., Watson, J. G., Kohl, S., Chen, L.-W. A., and Chai, W.: Measurements and validation for the 2008/2009 particulate matter study in Hong Kong, Hong Kong Environmental Protection Department, Hong Kong, 2010.

Dou, J., Lin, P., Kuang, B.-Y., and Yu, J. Z.: Reactive oxygen species production mediated by humic-like substances in atmospheric aerosols: Enhancement effects by pyridine, imidazole, and their derivatives, Environ. Sci. Technol., 49, 6457-6465, doi:10.1021/es5059378, 2015.

Engling, G., Carrico, C. M., Kreidenweis, S. M., Collett, J. L., Day, D. E., Malm, W. C., Lincoln, E., Hao, W. M., Iinuma, Y., and Herrmann, H.: Determination of levoglucosan in biomass combustion aerosol by high-performance anion-exchange chromatography with pulsed amperometric detection, Atmos. Environ., 40, 299-311, doi:10.1016/j.atmosenv.2005.12.069, 2006.

Graham, B., Mayol-Bracero, O. L., Guyon, P., Roberts, G. C., Decesari, S., Facchini, M. C., Artaxo, P., Maenhaut, W., Köll, P., and Andreae, M. O.: Water-soluble organic compounds in biomass burning aerosols over Amazonia 1. Characterization by NMR and GC-MS, J. Geophys. Res., 107, 8047, doi:10.1029/2001JD000336, 2002.

Huang, X. H., Bian, Q., Ng, W. M., Louie, P. K., and Yu, J. Z.: Characterization of $\mathrm{PM}^{2.5}$ major components and source investigation in suburban Hong Kong: A one year monitoring study, Aerosol. Air. Qual. Res., 14, 237-250, doi:10.4209/aaqr.2013.01.0020, 2014.

Kuang, B. Y., Lin, P., Huang, X. H. H., and Yu, J. Z.: Sources of humic-like substances in the Pearl River Delta, China: positive matrix factorization analysis of $\mathrm{PM}^{2.5}$ major components and source markers, Atmos. Chem. Phys., 15, 1995-2008, doi:10.5194/acp-15-1995-2015, 2015.

Lin, P., Engling, G., and Yu, J. Z.: Humic-like substances in fresh emissions of rice straw burning and in ambient aerosols in the Pearl River Delta Region, China, Atmos. Chem. Phys., 10, 64876500, doi:10.5194/acp-10-6487-2010, 2010.

Malm, W. C., Sisler, J. F., Huffman, D., Eldred, R. A., and Cahill, T. A.: Spatial and seasonal trends in particle concentration and optical extinction in the United-States, J. Geophys. Res., 99, 1347=1370, 1994.

Murphy, M. J., Hillenbrand, L. J., Trayser, D., and Wasser, J.: Assessment of diesel particulate control - direct and catalytic oxidation, SAE Technical Paper 0148-7191, 1981.
Panteliadis, P., Hafkenscheid, T., Cary, B., Diapouli, E., Fischer, A., Favez, O., Quincey, P., Viana, M., Hitzenberger, R., Vecchi, R., Saraga, D., Sciare, J., Jaffrezo, J. L., John, A., Schwarz, J., Giannoni, M., Novak, J., Karanasiou, A., Fermo, P., and Maenhaut, W.: ECOC comparison exercise with identical thermal protocols after temperature offset correction - instrument diagnostics by in-depth evaluation of operational parameters, Atmos. Meas. Tech., 8, 779-792, doi:10.5194/amt-8-779-2015, 2015.

Piazzalunga, A., Bernardoni, V., Fermo, P., Valli, G., and Vecchi, R.: Technical Note: On the effect of water-soluble compounds removal on EC quantification by TOT analysis in urban aerosol samples, Atmos. Chem. Phys., 11, 10193-10203, doi:10.5194/acp-11-10193-2011, 2011.

Saylor, R. D., Edgerton, E. S., and Hartsell, B. E.: Linear regression techniques for use in the EC tracer method of secondary organic aerosol estimation, Atmos. Environ., 40, 7546-7556, doi:10.1016/j.atmosenv.2006.07.018, 2006.

Shi, Y., Ji, Y., Sun, H., Hui, F., Hu, J., Wu, Y., Fang, J., Lin, H., Wang, J., Duan, H., and Lanza, M.: Nanoscale characterization of $\mathrm{PM}_{2.5}$ airborne pollutants reveals high adhesiveness and aggregation capability of soot particles, Sci. Rep., 5, 11232, doi:10.1038/srep11232, 2015.

Subramanian, R., Khlystov, A. Y., and Robinson, A. L.: Effect of peak inert-mode temperature on elemental carbon measured using thermal-optical analysis, Aerosol. Sci. Technol., 40, 763780, doi:10.1080/02786820600714403, 2006.

Sullivan, A. P., Weber, R. J., Clements, A. L., Turner, J. R., Bae, M. S., and Schauer, J. J.: A method for on-line measurement of water-soluble organic carbon in ambient aerosol particles: Results from an urban site, Geophys. Res. Lett., 31, L13105, doi:10.1029/2004g1019681, 2004.

Tie, X., Wu, D., and Brasseur, G.: Lung cancer mortality and exposure to atmospheric aerosol particles in Guangzhou, China, Atmos. Environ., 43, 2375-2377, doi:10.1016/j.atmosenv.2009.01.036, 2009.

Turpin, B. J. and Huntzicker, J. J.: Identification of Secondary Organic Aerosol Episodes and Quantitation of Primary and Secondary Organic Aerosol Concentrations during Scaqs, Atmos. Environ., 29, 3527-3544, doi:10.1016/1352-2310(94)00276-Q, 1995.

Wang, Y., Chung, A., and Paulson, S. E.: The effect of metal salts on quantification of elemental and organic carbon in diesel exhaust particles using thermal-optical evolved gas analysis, Atmos. Chem. Phys., 10, 11447-11457, doi:10.5194/acp-10-114472010, 2010.

Watson, J. G., Chow, J. C., and Chen, L.-W. A.: Summary of organic and elemental carbon/black carbon analysis methods and intercomparisons, Aerosol. Air. Qual. Res., 5, 65-102, 2005.

World Bank: East Asia's changing urban landscape: measuring a decade of spatial growth, World Bank, Washington, DC: World Bank, 2015.

$\mathrm{Wu}, \mathrm{C}$. and Yu, J. Z.: Determination of primary combustion source organic carbon-to-elemental carbon (OC / EC) ratio using ambient OC and EC measurements: secondary OC-EC correlation minimization method, Atmos. Chem. Phys., 16, 5453-5465, doi:10.5194/acp-16-5453-2016, 2016.

Wu, C., Ng, W. M., Huang, J., Wu, D., and Yu, J. Z.: Determination of Elemental and Organic Carbon in $\mathrm{PM}_{2.5}$ in the Pearl River Delta Region: Inter-Instrument (Sunset vs. DRI Model 2001 
Thermal/Optical Carbon Analyzer) and Inter-Protocol Comparisons (IMPROVE vs. ACE-Asia Protocol), Aerosol. Sci. Technol., 46, 610-621, doi:10.1080/02786826.2011.649313, 2012. 